\title{
Electromagnetic Field in Hybrid Quantum Plasmonic-Photonic Systems
}

\author{
Bernardo Barbiellini ${ }^{1,2, *}$, Subhabrata Das ${ }^{3}$, Venkatesan Renugopalakrishnan ${ }^{4,5}$ and \\ Ponniseril Somasundaran ${ }^{3}$ \\ 1 School of Engineering Science, Lappeenranta University of Technology, Lappeenranta 53851, Finland \\ 2 Physics Department, Northeastern University, Boston, MA 02115, USA \\ 3 Langmuir Center of Colloids and Interfaces, Columbia University in the City of New York, \\ New York, NY 10027, USA; sd2957@columbia.edu (S.D.); ps24@columbia.edu (P.S.) \\ 4 Department of Chemistry and Chemical Biology, Northeastern University, Boston, MA 02115, USA; \\ v.renugopalakrishnan@northeastern.edu \\ 5 Children's Hospital, Harvard Medical School, Boston, MA 02115, USA \\ * Correspondence: bernardo.barbiellini@lut.fi; Tel.: +358-50-310-5870
}

Received: 23 January 2018; Accepted: 27 March 2018; Published: 2 April 2018

\begin{abstract}
We study excitations and quantum optical properties of hybrid networks made up of metal nanoparticles, semiconductor quantum dots and molecules. Such processes can be used for the localization and the guiding of the electromagnetic field. Localized modes occurring in these networks and the generation of confined fields are also connected to the enhancement of Raman-scattering occurring in these systems. We review some recent theoretical and computational studies of optical properties in hybrid nano-systems to gain control of light-matter interactions at the quantum level for efficient energy transport and sensing applications.
\end{abstract}

Keywords: Forster resonance energy transfer (FRET); semiconductor quantum dots (SQDs); surface-enhanced Raman scattering (SERS)

\section{Introduction}

The electromagnetic field in vacuum and in homogeneous media can be expressed as the sum of plane monochromatic waves characterized by frequency and wavelength connected by velocity of light. Therefore, in these simple cases, a plane monochromatic wave is the elementary entity of the electromagnetic field. The corpuscular property of this wave is revealed by the existence of a quantum of energy proportional to the wave frequency. The proportionality factor is given by the Planck constant. Interestingly, this quantum of electromagnetic field (the photon) has characteristics of a particle, such as energy and momentum. In fact, several phenomena such as Compton and Raman scattering cannot be fully explained if this corpuscular aspect is neglected. The electromagnetic field in nano-structured systems made up of molecules, semiconductor quantum dots (SQDs) and metal nanoparticles (MNPs) is different from the field in vacuum or in homogeneous media, and resonant couplings with electronic levels and charge density oscillations lead to electromagnetic field confinement that can be used in optoelectronic applications. Light-matter interactions in metallic nanosystems are assisted by synchronized oscillation of electron charge called plasmons [1]. The new field of quantum plasmonics has opened up new frontiers for fundamental physics and realization of nanoscale quantum devices [2,3] by overcoming material limits for nano-photonic systems and nanolasers $[4,5]$. MNPs are also of great interest because their applications in chemical and biological sensors [6], cancer treatment [7], surface enhanced Raman spectroscopy (SERS) [8-10] and light harvesting [11]. The present paper reviews recent theoretical methods to describe and to quantize the 
electromagnetic field in these hybrid nano-structured systems. It also discusses SERS mechanisms and strong electromagnetic field confinement enabling single molecule spectroscopy [9].

\section{Design of Artificial Solids with Special Optical Properties}

Green plants transfer electromagnetic energy from chromophore to chromophore until it reaches the so-called reaction center where photosynthesis takes place [12]. This energy travels in the form of an exciton (electron-hole pair), a quantum state, which exhibits particle-wave duality; the aspect which predominates in a given system depends on how the exciton interacts with its surroundings and can be simulated using a positronium in a metal-organic framework [13]. For instance, an exciton placed in a periodic lattice behaves like an extended wave, while in the presence of disorder, the exciton can localize as a classical particle. Excitons can experience competing effects in plants: disorder and some interactions tend to localize individual excitons at chromophores while the energy resonant transfer tends to delocalize the excitons. These processes, including the cavity-enhanced transport of excitons [14], may play a crucial role in the new field of artificial photosynthesis [15], which has mobilized both scientists and engineers. Moreover, the coupling of organic semiconductors with plasmons can yield coherent states with higher conductivity and mobility [16-19]. Similar couplings [20] leads also to tunable work-functions in organic devices like light-emitting devices, solar cells and transistors [21,22]. Building blocks for artificial solids are presently available because of advances in nano-material synthesis, characterization, and understanding of their size-dependent properties. Nano-crystals (NCs) such as SQDs stand out as building blocks for the fundamental study of artificial solids and their technological development [23]. A very promising application are photovoltaic devices based on light harvesting layers composed of SQDs [24]. The adjustable parameters of artificial solids are: (1) the electronic structure of NCs as artificial atom by tailoring the quantum confinement of the electron wave function; (2) the inter-NC coupling for exciton dynamics; (3) the self-assembly of NCs into ordered superstructures as artificial crystals; and (4) the synthesis of hybrid systems [23]. Interestingly, in small NCs, surfaces and interfaces play a crucial role in energy transport. Moreover, artificial solids can facilitate the formation of an exciton Bose Einstein Condensate (BEC) with a spatial coherence build-up [25] and lasing properties [26]. Such systems can be also used to simulate efficient mechanisms for energy transfer in light harvesting systems [27].

In solar energy harvesting, one seeks to develop an absorbing layer to funnel the energy flow by a combination of electronic, optical and excitonic means $[17,18]$. This goal of efficient energy transfer could be achieved for example by designing a hybrid layer composed of SQDs and the photochromic protein bacteriorhodopsin (bR) as shown in Figure 1 [11,28]. Near-field resonance of electric dipoles drives this transfer in the SQD/bR layer, and this mechanism is generally known as Forster resonance energy transfer (FRET). The FRET rate and efficiency have been analyzed with respect to donor-acceptor species by King et al. [28] with comparable electronic energy gaps. In the model shown in Figure 2, the coupling elements SQD and bR are governed by point-dipole/point-dipole interaction.

The calculations use density matrix formalism linking the Perrin's coherent FRET model to the standard incoherent hopping dynamics in electronic energy transfer derived by Forster from the Fermi's golden rule. In order to introduce the vibronic coupling in $\mathrm{bR}$, one considers the line shape of a Lorentzian resonance. The width of the Lorentzian curve characterizes the coupling with the $\mathrm{bR}$ vibrations and determines the lifetime of the exciton. In the present scheme, the coupling with vibrations always produces de-coherence. This model is applicable in cases when the acceptor (bR) can vibrate while the donor molecule (SQD) is rigid. In this case, the model can be solved exactly [28]. The result shown in Figure 3 can be compared to the standard Forster incoherent limit. A striking difference with the standard Forster limit is the coherent oscillation in the time evolution of the donor exciton occupation shown in the lower panel of Figure 3. This feature appears when the width of the Lorentzian line shape is small. 


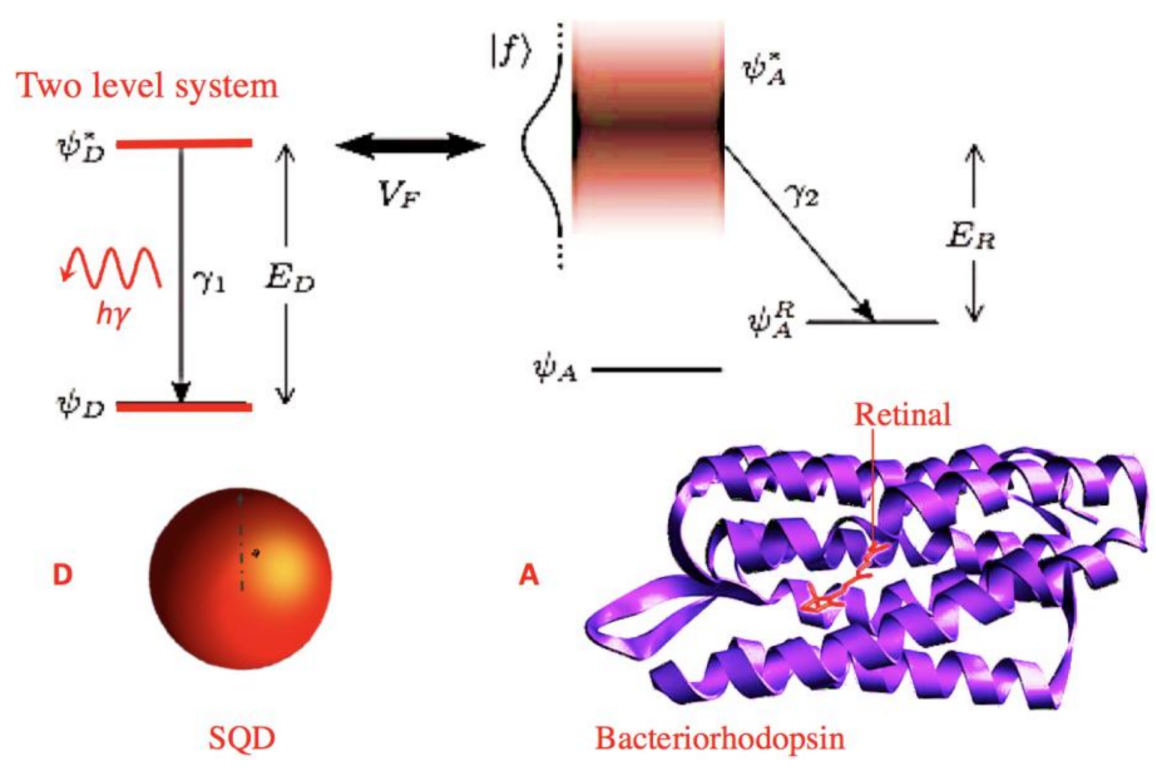

Figure 1. Model of CdSe quantum dot resonantly coupled to Bacteriorhodopsin from [28].

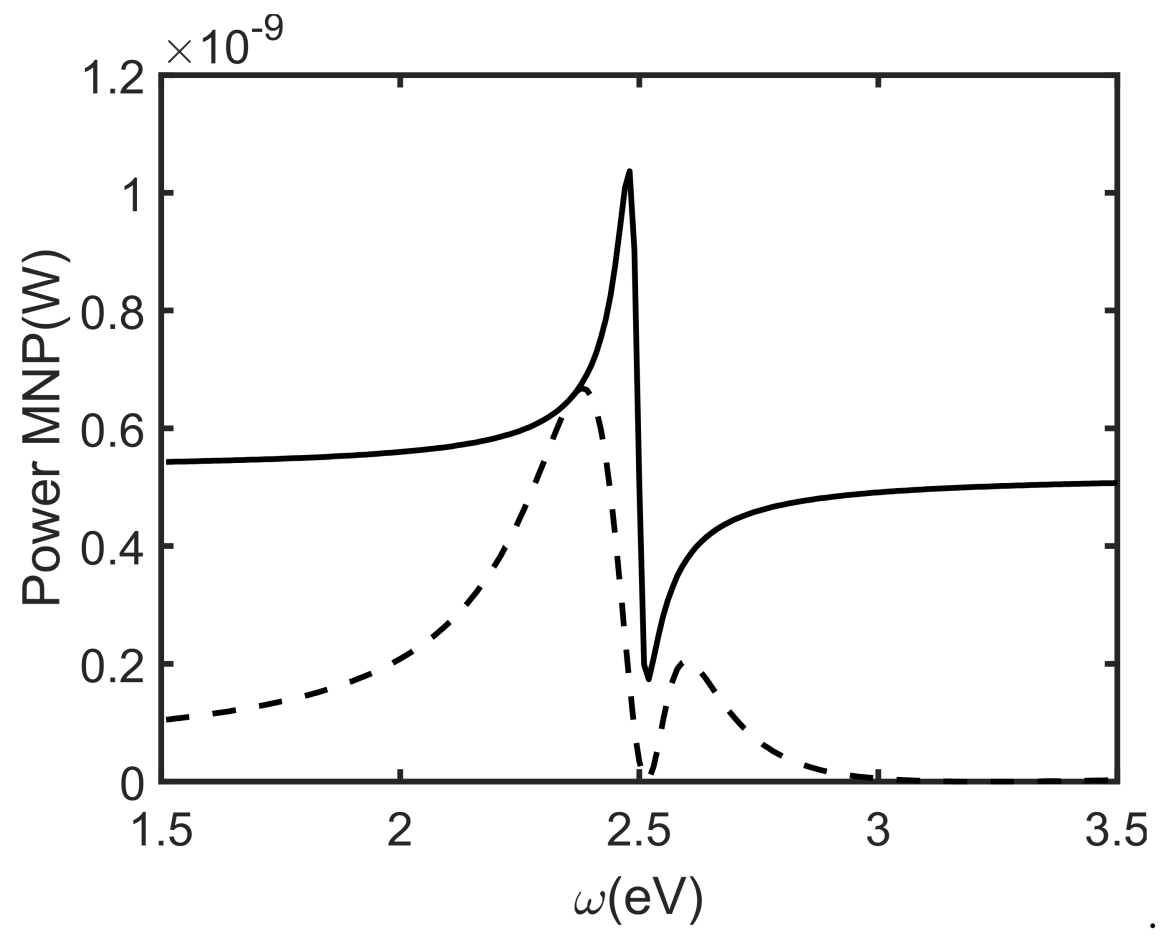

Figure 2. Absorption power of the metal nanoparticles (MNPs) for a SQD/MNP dimer case $[R=13 \mathrm{~nm}$ is the SQD-MNP distance; $\mathrm{a}=3 \mathrm{~nm}$ is the MNP radius and the light intensity is $10^{3} \mathrm{~W} / \mathrm{cm}^{2}$ ]; the solid line presents a Fano shape for an SQD electric dipole $\mu=0.25 \mathrm{e} \mathrm{nm}$, and the dashed line shows excitation induced transparency and a modified Fano shape, for an SQD electric dipole $\mu=2$ e nm. 

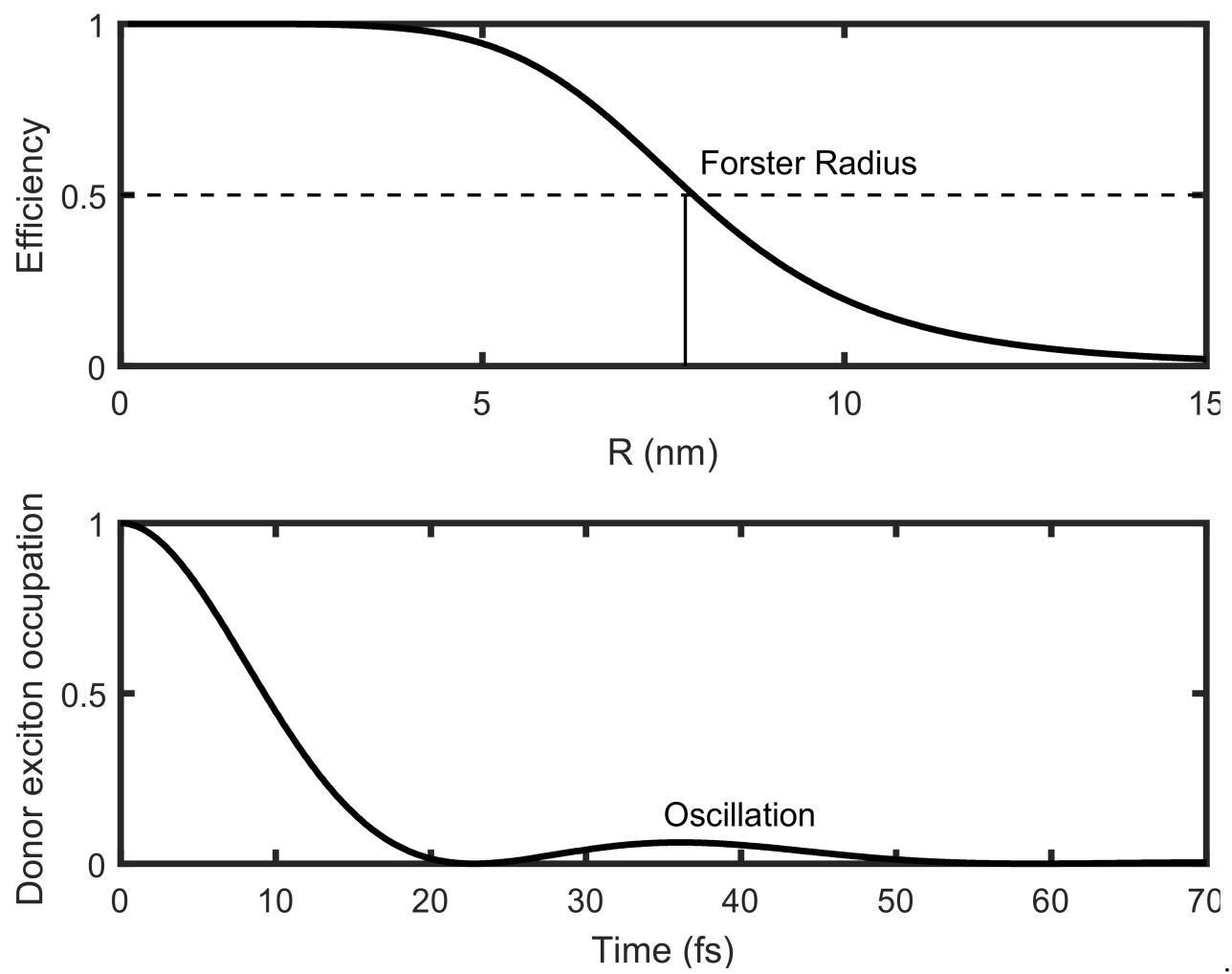

Figure 3. Results from the semiconductor quantum dot (SQD)/ bacteriorhodopsin (bR) model of [28]. Upper panel: Efficiency as function of distance. Lower panel: time evolution of the donor exciton occupation displaying a coherent oscillation.

First-principles simulations can provide parameters for the exact model by King et al. [28] yielding Forster radii for the engineered SQD/bR hybrid material [12]. Interestingly, recent experimental work [11] has suggested that the SQD/bR system can provide Forster radii significantly greater than those predicted by the standard incoherent hopping (without coherent oscillation) [28]. Among the several advantages of SQDs as photo-absorbers, their ability to tune the absorption spectrum by modifying their morphology and size is most significant [24]. Nevertheless, the re-emission of the absorbed light through fluorescence by the SQDs can result in photo-current losses and less efficient photovoltaic devices. The fluorescence emission of SQDs can be reduced if the SQD absorption layer is replaced by a bR/SQD hybrid layer [12]. In this architecture, the complexes are engineered in such a way that each QD is coupled with one bR trimer thus providing both spatial and optical $\mathrm{bR} / \mathrm{SQD}$ coupling. This design ensures maximal spectral overlap between donor fluorescence and acceptor absorption while maintaining minimal spatial distance between donor (SQD) and acceptor (bR). The maximal efficiency of resonance energy transfer in bR/SQD hybrid material emanates from the optical coupling between the SQD and bR, which directs re-emitted light from the SQD within its energy non-radiative transfer to $\mathrm{bR}$, thereby increasing the amount of trapped incident energy available for photoelectric conversion. This coupling, based on FRET, provides an efficient energy transfer pathway between molecules separated by distances of up to $15 \mathrm{~nm}$. King et al. [28] have shown that a correct treatment of quantum coherence plays a pivotal role for enlarging the Forster radius. Such quantum coherence is also important for charge separation efficiency in the Reaction Center Complexes (RCC)s of plant photosystem II [29]. Furthermore, a cis-trans isomerization induced conformational distortion in the retinal molecule helps bR to effectively lock the energy in place and thus act as an efficient trap for light energy. Thus, the bR acts as the storage device and the SQD as the antenna in bR/SQD hybrid networks. 


\section{Hybrid Plasmonic-SQD Platform}

The resonant coupling of MNPs to the SQD in the presence of an applied driving field has been extensively studied [30]. In particular, Hayati et al. [31] have considered an efficient approach based on the quantum density-matrix formalism, which yields Fano lines shapes [32]. The absorption power of a MNP of radius $\mathrm{a}=3 \mathrm{~nm}$ belonging to a SQD/MNP dimer is illustrated in Figure 2. The SQD-MNP distance is $\mathrm{R}=13 \mathrm{~nm}$ and the $2.5 \mathrm{eV}$ light intensity is $10^{3} \mathrm{~W} / \mathrm{cm}^{2}$. The solid curve presents an asymmetric Fano shape for an SQD electric dipole $\mu=0.25 \mathrm{e} \mathrm{nm}$ while the dashed line shows excitation induced transparency and a modified Fano shape, for an SQD electric dipole $\mu=2$ e nm. Clearly, the two curves are very different from a Lorentzian shape. In the present case, the standard density matrix time dependent treatment [33] is limited to a few building blocks since it considers a set of complex nonlinear ordinary differential equations (ODEs) as explained in [31]. In order to alleviate the computational bottleneck due to the system size, Hayati et al. have used a set of linear von Neumann equations of motion in the steady state for the density matrix of each SQD placed in an effective field calculated within the discrete dipole approximation [31]. In this regime, the method becomes scalable and its numerical efficiency allows the treatment of very large hybrid networks. Plasmonics components such as gold thin films can be also used to convert light in a current of electron by charge separation [11]. More specifically, resonant photons coupled to an optical gold nano-antenna relax into "hot", energetic electrons [34], which, when driven over the Schottky barrier formed by the nanoantenna/semiconductor interface, generate a photocurrent. In general, electrons and positive holes once formed in a dye or SQD absorbing layer must be kept several nanometers apart before the charge recombination between the electron and hole occurs. The basic idea of charge separation is to move electrons from one side of a potential barrier to the other side as quickly and as efficiently as possible, so that the positive and negative charges become spatially remote.

For example, the Auger mediated de-excitation (AMD) is an efficient quantum mechanism for charge separation by transferring the energy from the absorbing layer (donor) to a gold nano-antenna (acceptor) through a single step and it is similar to a photoelectric effect involving virtual photons. Since only virtual (i.e., non-real) photons are involved in the AMD process, the selection rules do not apply, therefore the rates are large and thus the injection times are short. Near-field electrodynamics permits energy transfer without real photon emission and a particular case of AMD is given by the Auger mediated sticking (AMS) of a positron colliding against a metallic surface [35]. As illustrated in Figure 4, the positron transitions from a scattering state to a metal surface state and the excess of energy is transferred to a valence electron emitted in the vacuum. Such non-radiative decay phenomenon is of great scientific interest in different areas of physics and chemistry [36-38]. For instance, new designs for solar energy conversion use non-radiative mechanisms for the energy transfer from excitons created on a photon absorption layer to high mobility charge carriers inside the solar cell. This transfer relies on the near-field resonance of electric dipoles and it is similar to the FRET coupling discussed in Section 2. 


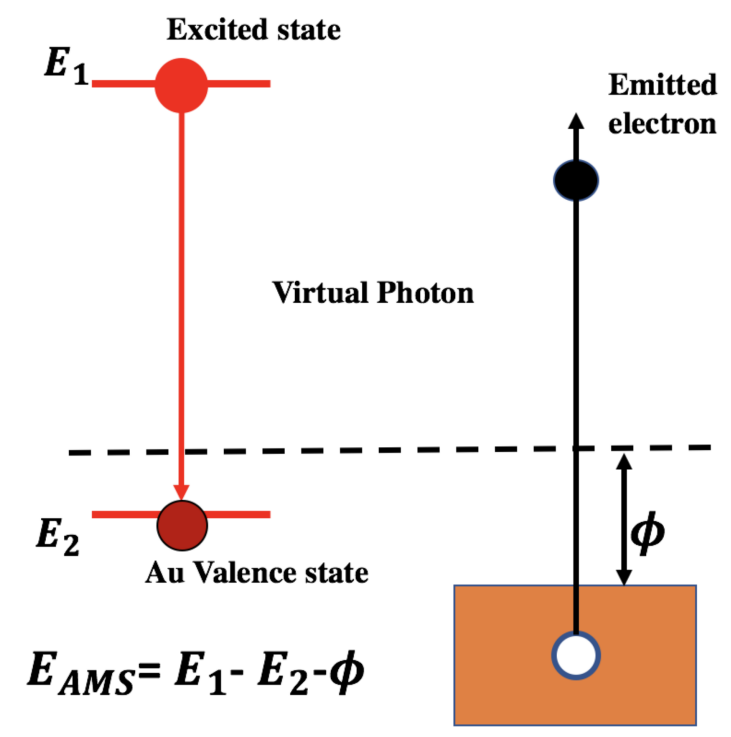

Figure 4. Schematics of the Auger mediated sticking (AMS) effect: The dotted line indicates the vacuum level. Copper is a typical example with work function of about $\phi=4.5 \mathrm{eV}$ and a positron bound state $\mathrm{E}_{2}=-2.8 \mathrm{eV}$. $\mathrm{E}_{\mathrm{AMS}}$ is the kinetic energy of the emitted electron.

\section{SERS and Electromagnetic Field Localization}

Spontaneous emission is a genuine quantum process, since the excited state of an atom cannot decay into the ground state without a quantized electromagnetic field [39]. This process can be explained only if the electromagnetic field is quantized. It is also important to keep in mind that spontaneous emission is not an inherent characteristic of the emitter, but it depends on the local electromagnetic environment. Interestingly, chemical reactions as spontaneous emission can also be affected by the local electromagnetic environment [40]. A useful point of view to understand the modification of spontaneous emission by MNPs can rely on the FRET language. As explained in Section 2, two emitters can exchange energy through FRET. In a particular example of modified spontaneous emission provided by Pelton [41], a MNP replaces the second emitter. As a result, the FRET coupling modifies the spontaneous emission of the SQD, which is the first emitter. This mechanism is also known as the Purcell effect. It can produce a 540-fold increase of the emission decay rate and a 1900-fold enhancement of total emission intensity for SQDs coupled to a plasmonic nanocavity [42]. Similar manipulations of the emission decay rate can be useful for wireless communication between devices using light to transmit data [43]. As in modified spontaneous emission, changes in SERS are produced by the interaction of the molecules with MNP plasmon resonances. Nevertheless, the explanation of SERS at molecules on Au or Ag metal surfaces is a more challenging [44] than the explanation of the Purcell effect. Electromagnetism is clearly the driving force behind SERS and the electronic structure of $\mathrm{Au}$ or $\mathrm{Ag}$ also plays an important role [45]. To tackle this difficult problem, a Renormalization Group (RG) approach beyond perturbation theory has been considered [46]. In this framework, the valence electrons of a molecule are embedded in an effective medium with dielectric function $\varepsilon$, which integrates out the effect of the plasmonic excitations of MNPs. By using a thermodynamic argument [47], one can show that the effective classical radius of the valence electrons in the molecule is renormalized as follows

$$
\mathrm{r}_{0}{ }^{*}=\exp \left[\left(\varepsilon_{1} / \varepsilon_{0}-1\right) /(3 \alpha)\right] \mathrm{r}_{0}
$$

where $\varepsilon_{0}$ is the vacuum dielectric constant, $\alpha=1 / 137$ is the fine structure constant (i.e., the effective coupling when $\varepsilon=\varepsilon_{0}$ ) while $\varepsilon_{1}$ is the real part of an effective dielectric function $\varepsilon$. The bare classical radius of the electron $r_{0}$, has a very small size given by $2.82 \times 10^{-15} \mathrm{~m}$. The effective cross section 
of a valence electron scattered by a photon is proportional to the square of $r_{0}{ }^{*}$. Therefore, there are anomalous dispersions near the plasmonic absorption edges, that allow the Raman scattering cross section to be enhanced in the domain where $\varepsilon_{1} / \varepsilon_{0}-1$ is positive. Interestingly, $\varepsilon_{1} / \varepsilon_{0}=1.3$ can explain observed enhancements of 14 orders of magnitude [9]. A typical example of such a situation is the single crystal violet molecule detection by SERS in a colloidal silver solution [48]. That means that the ratio $\mathrm{r}_{0}{ }^{*} / \mathrm{r}_{0}$ could be of the order of $10^{7}$ and therefore the scale of $\mathrm{r}_{0}{ }^{*}$ is about $100 \mathrm{~nm}$. Clearly $\varepsilon_{1} / \varepsilon_{0}>1$ is an important condition for the resonant enhancement of the stimulated electronic Raman Scattering. In fact, when $\varepsilon_{1} / \varepsilon_{0}$ is smaller than one, the cross section weakens instead of growing. In resonant X-ray inelastic scattering $[47,49,50], \varepsilon_{1}$ can be evaluated by using first-principles quantum calculations but for SERS the ab initio determination of $\varepsilon_{1}$ is still lacking. Therefore, the connection with first-principles calculations has to be established to verify the present model. We turn now to the discussion of the electromagnetic field confinement in the SERS process. According to Mael Melvin [51], an electronic charge distribution described by a renormalized $\mathrm{r}_{0}{ }^{*}$ and coupled to a classical electromagnetic field leads to an analogue of the London penetration depth for the electromagnetic field that can be expressed as

$$
L=\mathrm{r}_{\mathrm{S}}\left[4 \pi / 3 \mathrm{r}_{\mathrm{s}} / \mathrm{r}_{0}{ }^{*}\right]^{1 / 2},
$$

where the length $r_{s}$ represents the radius containing a valence electron. Charles Enz has derived a similar confinement length $L$ for the electromagnetic field and he has also quantized the corresponding electromagnetic field [52]. Therefore, $L$ can be viewed as the inverse of an effective mass of the photon in the system. Effective massive photons in a medium have also been recently discussed by Arbab $[53,54]$. Here, the length $L$ can lead to a large mass and a strong confinement. If we assume that $\mathrm{r}_{\mathrm{s}}$ about $1 \mathrm{~nm}$, then $L$ becomes of the order of a tenth of nanometer. Thus, SERS can be transformed into a single molecule spectroscopic probe, that can compress the electromagnetic field at the molecular scale [9].

\section{Conclusions}

Optoelectronics hybrid nano-networks can be studied by combining Quantum Mechanics and Maxwell's equations. This area of research is nowadays very active and typical examples from recent research include studies of metamaterials with unusual optical properties [55-58], bifunctional nanocomposites probed by SERS [59] and generation of entangled photons [60]. In this short review, we have focused on conceptual advances for FRET, modified spontaneous emission and SERS. Progress has been made, but the field must still converge to a more unified description since the corpuscular behavior of light needed to fully explain both the Compton and Raman effects is often neglected. To address this problem, a preliminary RG approach has been proposed. This scheme explains large Raman scattering cross sections from molecules on surfaces of metallic nanoparticles and strong confinements of the electromagnetic field. The present theoretical efforts can lead to paradigm shifts needed for optoelectronics nanoscale applications such as efficient energy transport, wave guiding and sensing [61].

Acknowledgments: B.B. benefitted form support of the U.S. Department of Energy, Office of Science, Basic Energy Sciences contract no. DE-FG02-07ER46352, and he has benefited from Northeastern University's Advanced Scientific Computation Center (ASCC), theory support at the Advanced Light Source, Berkeley, and the allocation of supercomputer time at NERSC through grant no. DE-AC02-05CH11231. V.R. acknowledges partial support from NIH, NSF, Rothschild Foundation, and Harvard Medical School.

Author Contributions: B.B. conceived the problem statement based on his current work and work of other groups. B.B. and S.D. wrote the manuscript. V.R. and P.S. provided critical feedback to the manuscript based on the current work in SERS. All the authors revised the manuscript.

Conflicts of Interest: The authors declare no conflict of interest. 


\section{References}

1. Novotny, L.; van Hulst, N. Antennas for light. Nat. Photonics 2011, 5, 83-90. [CrossRef]

2. Tame, M.S.; McEnery, K.R.; Özdemir, Ş.K.; Lee, J.; Maier, S.A.; Kim, M.S. Quantum plasmonics. Nat. Phys. 2013, 9, 329. [CrossRef]

3. Cushing, S.K.; Li, J.; Meng, F.; Senty, T.R.; Suri, S.; Zhi, M.J.; Li, M.; Bristow, A.D.; Wu, N.Q. Photocatalytic activity enhanced by plasmonic resonant energy transfer from metal to semiconductor. J. Am. Chem. Soc. 2012, 134, 15033-15041. [CrossRef] [PubMed]

4. Zhang, C.; Kinsey, N.; Chen, L.; Ji, C.G.; Xu, M.J.; Ferrera, M.; Pan, X.; Shalaev, V.M.; Boltasseva, A.; Guo, L.J. High-performance doped silver films: Overcoming fundamental material limits for nanophotonic applications. Adv. Mater. 2017, 29, 1605177. [CrossRef] [PubMed]

5. Noginov, M.A. Demonstration of a spaser-based nanolaser. Nature 2009, 460, 1110-1112. [CrossRef] [PubMed]

6. Yao, Y.; Yi, B.; Xiao, J.; Li, Z. Surface Plasmon resonance biosensors and its application. In Proceedings of the 2007 1st International Conference on Bioinformatics and Biomedical Engineering, Wuhan, China, 6-8 July 2007; pp. 1043-1046.

7. Szunerits, S.; Boukherroub, R. Sensing using localised surface plasmon resonance sensors. Chem. Commun. 2012, 48, 8999-9010. [CrossRef] [PubMed]

8. Nie, S.; Emory, S.R. Probing single molecules and single nanoparticles by surface-enhanced raman scattering. Science 1997, 275, 1102. [CrossRef] [PubMed]

9. Kneipp, K. Surface-enhanced raman scattering. Phys. Today 2007, 60, 40. [CrossRef]

10. Wang, Y.; Irudayaraj, J. Surface-enhanced raman spectroscopy at single-molecule scale and its implications in biology. Philos. Trans. R. Soc. B Biol. Sci. 2013, 368, 20120026. [CrossRef] [PubMed]

11. Renugopalakrishnan, V.; Barbiellini, B.; King, C.; Molinari, M.; Mochalov, K.; Sukhanova, A.; Nabiev, I.; Fojan, P.; Tuller, H.L.; Chin, M. Engineering a robust photovoltaic device with quantum dots and bacteriorhodopsin. J. Phys. Chem. C 2014, 118, 16710-16717. [CrossRef] [PubMed]

12. Somasundaran, P.; Chin, M.; Latosiewicz, U.T.; Tuller, H.L.; Barbiellini, B.; Renugopalakrishnan, V. Nanoscience and Engineering for Robust Biosolar Cells; CRC Press: Boca Raton, FL, USA, 2011.

13. Crivelli, P.; Cooke, D.; Barbiellini, B.; Brown, B.L.; Feldblyum, J.I.; Guo, P.; Gidley, D.W.; Gerchow, L.; Matzger, A.J. Positronium emission spectra from self-assembled metal-organic frameworks. Phys. Rev. B 2014, 89, 241103. [CrossRef]

14. Schachenmayer, J.; Genes, C.; Tignone, E.; Pupillo, G. Cavity-enhanced transport of excitons. Phys. Rev. Lett. 2015, 114, 196403. [CrossRef] [PubMed]

15. Benniston, A.C.; Harriman, A. Artificial photosynthesis. Mater. Today 2008, 11, 26-34. [CrossRef]

16. Orgui, E.; George, J.; Hutchison, J.A.; Devaux, E.; Dayen, J.F.; Doudin, B.; Stellacci, F.; Genet, C.; Schachenmayer, J.; Genes, C.; et al. Conductivity in organic semiconductors hybridized with the vacuum field. Nat. Mater. 2015, 14, 1123-1129. [CrossRef] [PubMed]

17. Lidzey, D.G.; Bradley, D.D.C.; Skolnick, M.S.; Virgili, T.; Walker, S.; Whittaker, D.M. Strong excitonphoton coupling in an organic semiconductor microcavity. Nature 1998, 395, 53-55. [CrossRef]

18. Dintinger, J.; Klein, S.; Bustos, F.; Barnes, W.L.; Ebbesen, T.W. Strong coupling between surface plasmon-polaritons and organic molecules in subwavelength hole arrays. Phy. Rev. B 2005, 71, 035424. [CrossRef]

19. Bellessa, J.; Bonnand, C.; Plenet, J.C.; Mugnier, J. Strong coupling between surface plasmons and excitons in an organic semiconductor. Phys. Rev. Lett. 2004, 93, 03604. [CrossRef] [PubMed]

20. Schwarz, T.; Hutchison, J.A.; Genet, C.; Ebbesen, T.W. Reversible switching of ultrastrong light-molecule coupling. Phys. Rev. Lett. 2011, 106, 196405. [CrossRef] [PubMed]

21. Hutchison, J.A. Tuning the work-Function via strong coupling. Adv. Mater. 2013, 25, 2481-2485. [CrossRef] [PubMed]

22. Feist, J.; Garcia-Vidal, F.J. Extraordinary exciton conductance induced by strong coupling. Phys. Rev. Lett. 2015, 114, 196402. [CrossRef] [PubMed]

23. Agranovich, V.M.; Gartstein, Y.N.; Litinskaya, M. Hybrid resonant organic-inorganic nanostructures for optoelectronic applications. Chem. Rev. 2011, 111, 5179-5214. [CrossRef] [PubMed]

24. Kamat, P.V. Quantum dot solar cells. Semiconductor nanocrystals as light harvesters. J. Phys. Chem. C 2008, 112, 18737-18753. [CrossRef] 
25. Kasprzak, J.; Richard, M.; Kundermann, S.; Baas, A.; Jeambrun, P.; Keeling, J.M.J.; Marchetti, F.M.; Szymańska, M.H.; André, R.; Staehli, J.L.; et al. Bose-Einstein condensation of exciton polaritons. Nature 2006, 443, 409-414. [CrossRef] [PubMed]

26. Kéna-Cohen, S.; Forrest, S.R. Room-temperature polariton lasing in an organic single-crystal microcavity. Nat. Photonics 2010, 4, 371-375. [CrossRef]

27. Coles, D.M.; Somaschi, N.; Michetti, P.; Clark, C.; Lagoudakis, P.G.; Savvidis, P.G.; Lidzey, D. Polariton-mediated energy transfer between organic dyes in a strongly coupled optical microcavity. Nat. Mater. 2014, 13, 712-719. [CrossRef] [PubMed]

28. King, C.; Barbiellini, B.; Moser, D.; Renugopalakrishnan, V. Exactly soluble model of resonant energy transfer between molecules. Phys. Rev. B 2012, 85, 125106. [CrossRef]

29. Romero, E.; Augulis, R.; Grondelle, R.V. Quantum coherence in photosynthesis for efficient solar-energy conversion. Nat. Phys. 2014, 10, 676-682. [CrossRef] [PubMed]

30. Cao, E.; Lin, W.; Sun, M.; Liang, W.; Song, Y. Exciton-plasmon coupling interactions: From principle to applications. Nanophotonics 2018, 7, 145. [CrossRef]

31. Hayati, L.; Lane, C.; Barbiellini, B.; Bansil, A.; Mosallaei, H. Self-consistent scheme for optical response of large hybrid networks of semiconductor quantum dots and plasmonic metal nanoparticles. Phys. Rev. B 2016, 93, 245411. [CrossRef]

32. Miroshnichenko, A.E.; Flach, S.; Kivshar, Y.S. Fano resonances in nanoscale structures. Rev. Mod. Phys. 2010, 82, 2257-2298. [CrossRef]

33. Artuso, R.D.; Bryant, G.W. Optical response of strongly coupled quantum dot-metal nanoparticle systems: Double peaked fano structure and bistability. Nano Lett. 2008, 8, 2106-2111. [CrossRef] [PubMed]

34. Harutyunan, H.; Martinson, A.B.F.; Rosenmann, D.; Khorashad, L.K.; Besteiro, L.V.; Govorov, A.O.; Wiederrecht, G.P. Anomalous ultrafast dynamics of hot plasmonic electrons in nanostructures with hot spots. Nat. Nanotechnol. 2015, 10, 770-774. [CrossRef] [PubMed]

35. Mukherjee, S.; Nadesalingam, M.P.; Guagliardo, P.; Sergeant, A.D.; Barbiellini, B.; Williams, J.F.; Fazleev, N.G.; Weiss, A.H. Auger-mediated sticking of positrons to surfaces: Evidence for a single-step transition from a scattering state to a surface image potential bound state. Phys. Rev. Lett. 2010, 104, 247403. [CrossRef] [PubMed]

36. Barbiellini, B.; Platzman, P.M. The healing mechanism for excited molecules near metallic surfaces. New J. Phys. 2006, 8, 20. [CrossRef]

37. Vittorini-Orgeas, A.; Bianconi, A. From Majorana theory of atomic autoionization to Feshbach resonances in high temperature superconductors. J. Supercond. Nov. Magn. 2009, 22, 215-221. [CrossRef]

38. Chirayath, V.A.; Callewaert, V.; Fairchild, A.J.; Chrysler, M.D.; Gladen, R.W.; Mcdonald, A.D.; Imam, S.K.; Shastry, K.; Koymen, A.R.; Saniz, R.; et al. Auger electron emission initiated by the creation of valence-band holes in graphene by positron annihilation. Nat. commun. 2017, 8, 16116. [CrossRef] [PubMed]

39. Novotny, L.; Hecht, B. Principles of Nano-optics; Cambridge University Press: London, UK, 2012.

40. Hutchison, J.A. Modifying chemical landscapes by coupling to vacuum fields. Angew. Chem. 2012, 51, 1592-1596. [CrossRef] [PubMed]

41. Pelton, M. Modified spontaneous emission in nanophotonic structures. Nat. Photonics 2015, 9, 427-435. [CrossRef]

42. Hoang, T.B.; Akselrod, G.M.; Mikkelsen, M.H. Ultrafast room-temperature single photon emission from quantum dots coupled to plasmonic nanocavities. Nano Lett. 2016, 16, 270-275. [CrossRef] [PubMed]

43. Lu, Y.-J.; Sokhoyan, R.; Cheng, W.-H.; Shirmanesh, G.K.; Davoyan, A.R.; Pala, R.A.; Thyagarajan, K.; Atwater, H.A. Dynamically controlled Purcell enhancement of visible spontaneous emission in a gated plasmonic heterostructure. Nat. Commun. 2017, 8, 1631. [CrossRef] [PubMed]

44. Masiello, D.J. Multiscale theory and simulation of plasmon-enhanced molecular optical processes. Int. J. Quantum Chem. 2014, 114, 1413-1420. [CrossRef]

45. Kediziora, G.; Schatz, G. Calculating dipole and quadrupole polarizabilities relevant to surface enhanced Raman spectroscopy. Spectrochim. Acta Part A 1999, 55, 625-638. [CrossRef]

46. Barbiellini, B. Enhancement of Raman scattering from molecules placed near metal nanoparticles. Low Temp. Phys. 2017, 43, 159-161. [CrossRef]

47. Barbiellini, B.; Nicolini, P. Enhancement of Compton scattering by an effective coupling constant. Phys. Rev. A 2011, 84, 22509. [CrossRef] 
48. Kneipp, K.; Wang, Y.; Kneipp, H.; Perelman, L.T.; Itzkan, I.; Dasari, R.R.; Feld, M.S. Single molecule detection using surface-enhanced Raman scattering (SERS). Phys. Rev. Lett. 1996, 78, 1667. [CrossRef]

49. Barbiellini, B.; Hancock, J.N.; Monney, C.; Joly, Y.; Ghiringhelli, G.; Braicovich, L.; Schmitt, T. Inelastic X-ray scattering from valence electrons near absorption edges of FeTe and TiSe 2 . Phys. Rev. B 2014, 89, 235138. [CrossRef]

50. Barbiellini, B.; Joly, Y.; Tamasaku, K. Explaining the X-ray nonlinear susceptibility of diamond and silicon near absorption edges. Phys. Rev. B 2015, 92, 155119. [CrossRef]

51. Melvin, M.A. The new classical electrodynamics. Nature 1953, 171, 890. [CrossRef]

52. Enz, C.P. On Preparata's theory of a superradiant phase transition. Helv. Phys. Acta 1997, 70, 141-153.

53. Arbab, A.I. On the refractive index and photon mass. Opt. Int. J. Light Electron Opt. 2016, 127, $6682-6687$. [CrossRef]

54. Arbab, A.I. On Compton scattering in dielectric medium. Opt. Int. J. Light Electron Opt. 2016, 127, 8536-8541. [CrossRef]

55. Lee, J.; Tymchenko, M.; Argyropoulos, C.; Chen, P.Y.; Lu, F.; Demmerle, F.; Boehm, G.; Amann, M.-C.; Alù, A.; Belkin, M.A. Giant nonlinear response from plasmonic metasurfaces coupled to intersubband transitions. Nature 2014, 511, 65-69. [CrossRef] [PubMed]

56. Wolf, O.; Campione, S.; Benz, A.; Ravikumar, A.P.; Liu, S.; Luk, T.S.; Kadlec, E.A.; Shaner, E.A.; Klem, J.F.; Sinclair, M.B.; et al. Phased-array sources based on nonlinear metamaterial nanocavities. Nat. Commun. 2015, 6, 7667. [CrossRef] [PubMed]

57. Wuestner, S.; Pusch, A.; Tsakmakidis, K.L.; Hamm, J.M.; Hess, O. Gain and plasmon dynamics in active negative-index metamaterials. Philos. Trans. R. Soc. A 2011, 369, 3525-3550. [CrossRef] [PubMed]

58. Rose, A.; Huang, D.; Smith, D.R. Controlling the second harmonic in a phase-matched negative-index metamaterial. Phys. Rev. Lett. 2011, 107, 063902. [CrossRef] [PubMed]

59. Yang, J.-L.; Xu, J.; Ren, H.; Sun, L.; Xu, Q.-C.; Zhang, H.; Li, J.-F.; Tian, Z.-Q. In situ SERS study of surface plasmon resonance enhanced photocatalytic reactions using bifunctional Au@CdS core-shell nanocomposites. Nanoscale 2017, 9, 6254-6258. [CrossRef] [PubMed]

60. Suchowsk, H.; O’Brien, K.; Wong, Z.J.; Salandrino, A.; Yin, X.; Zhang, X. Phase mismatch-free nonlinear propagation in optical zero-index materials. Science 2013, 342, 1223-1226. [CrossRef] [PubMed]

61. Noubir, G.; Barbiellini, B. Biologically-Enabled Wireless Networks Design and Modeling; National Science Foundation: Alexandria, VA, USA, 2011. 